\title{
Distribution of values of Hecke characters of infinite order
}

\author{
by
}

\author{
C. S. RAJAN (Mumbai)
}

We show that the number of primes of a number field $K$ of norm at most $x$, at which the local component of an idele class character of infinite order is principal, is bounded by $O(x \exp (-c \sqrt{\log x}))$ as $x \rightarrow \infty$, for some absolute constant $c>0$ depending only on $K$.

0. Introduction. The prime number theorem of Hadamard and de la Vallée Poussin states that the number $\pi(x)$ of rational primes less than $x$, grows as

$$
\pi(x)=\operatorname{li} x+O(x \exp (-c \sqrt{\log x}))
$$

for some suitable constant $c>0$. Our main result is to show that for idele class characters of infinite order, we can obtain a similar explicit "error term", concerning the distribution of the values of the character. More precisely, let $K$ be a number field and let $J_{K}$ (resp. $\mathbf{A}_{K}$ ) denote the group of ideles (resp. adeles) of $K$. Let $C_{K}=J_{K} / K^{*}$, be the idele class group of $K$. Let $\theta$ be a character of infinite order on $C_{K}$. For a finite place $v$ of $K$, denote by $K_{v}$ the completion of $K$ with respect to $v, N v$ the norm of $v$, and $\theta_{v}$ the $v$-component of $\theta$. Let $M_{f}$ denote the set of finite places of $K$. We show that for some constant $c>0$ depending only on $K$, and $x$ large enough, the following bound holds:

$$
\#\left\{v \in M_{f} \mid N v<x, \theta_{v}=1\right\}=O(x \exp (-c \sqrt{\log x})),
$$

where the implied constant depends only on $K$. Under the assumption of the Riemann Hypothesis for $L\left(s, \theta^{k}\right), k \geq 1$, we obtain the following LangTrotter type upper bound:

$$
\#\left\{v \in M_{f} \mid N v<x, \theta_{v}=1\right\}=O\left(x^{1 / 2}(\log x)^{2}\right) .
$$

We remark that Lang-Trotter type upper bounds of the form $O\left(x^{1 / 2+\varepsilon}\right)$ are not available for modular forms, even under the assumption of the Riemann Hypothesis [VKM].

1991 Mathematics Subject Classification: Primary 11R45; Secondary 11M41, 11N99. 
Hecke $[\mathrm{He}]$ showed the equidistribution of the values of $\theta$, provided $\theta$ is not equivalent to a Dirichlet character. In particular, he showed that $\#\left\{v \mid N v<x, \theta_{v}=1\right\}=o(x / \log x)$. Our result is a refinement of Hecke's theorem, with an explicit bound for the error term, which is similar to the error term occurring in the prime number theorem.

The proof can be considered as an application of the principle of Langlands-Rankin-Selberg, which asserts that the size of the Fourier coefficients of an automorphic representation can be controlled, provided it is possible to control the analytic behavior of the $L$-functions attached to the symmetric powers of the representation. In our case, the necessary analytic properties were established by Hecke. The proof of the prime number theorem, with the explicit error term as above, uses the explicit formula, together with a knowledge of the zeros of the Riemann zeta function. However, on applying the explicit formula method to the $L$-function $L(s, \theta)$ of $\theta$, we obtain an estimate for $\sum_{N v<x} \theta_{v}\left(\pi_{v}\right)$. In order to obtain the theorem, the idea is to apply the explicit formula method to the functions $L\left(s, \theta^{k}\right)$, for all positive integers $k$, and estimate sums of the form $\sum_{N v<x} \theta_{v}\left(\pi_{v}\right)^{k}$. We then invoke a result of Erdős-Turán on the discrepancy of a sequence, which can be considered as a quantitative analogue of the theorems on uniform distribution, to conclude the proof of our theorem.

The idea for the theorem arose from [MR], where we considered pairs of irreducible, unitary, cuspidal automorphic representations $\pi_{1}$ and $\pi_{2}$ on $G L_{2}\left(\mathbf{A}_{K}\right)$. Under the assumption that the functions $L\left(s, \operatorname{Sym}^{m}\left(\pi_{1}\right) \times\right.$ $\left.\operatorname{Sym}^{n}\left(\pi_{2}\right)\right)$ are entire, possess a functional equation of the appropriate kind, and satisfy the Generalised Riemann Hypothesis, we established the following:

$$
\#\left\{p \leq x \mid \pi_{1, v}=\pi_{2, v}\right\}=O\left(x^{5 / 6+\varepsilon}\right) .
$$

Acknowledgements. It is a pleasure to thank M. Ram Murty and V. Kumar Murty for their useful suggestions and discussions. In particular, I thank V. Kumar Murty and the referee for pointing out that the estimate in Proposition 4 can be improved to obtain Lang-Trotter type estimates. Kumar Murty informed me that he had obtained this result independently. I am also indebted to my colleagues at McGill University and CICMA for their pleasant hospitality and support during my stay there, in which period this work was done.

1. Analytic theory of $L(s, \theta)$. Let $K$ be a number field of degree $d$ over $\mathbb{Q}$. For a finite prime $v$, let $\mathcal{O}_{v}$ denote the ring of $v$-adic integers in $K_{v}$, and $U_{v}$ denote the group of $v$-adic units. Let $\mathfrak{p}_{v}$ be the maximal ideal of $\mathcal{O}_{v}$, and $\pi_{v}$ be a local uniformising parameter at $v$. The groups $U_{v}^{(m)}=\left(1+\mathfrak{p}_{v}^{m}\right)$, for $m$ a positive integer, form a decreasing filtration on $U_{v}$. We set $U_{v}^{(0)}=U_{v}$. 
The exponent $f_{v}=f_{v}(\theta)$ of the conductor of $\theta$ at $v$ is defined to be the least $m$ such that $\theta_{v}$ is trivial on $1+\mathfrak{p}_{v}^{m}$. $\theta$ is unramified at $v$ if $f_{v}(\theta)=0$. Since there are no small subgroups in $\mathbb{C}^{*}, \theta$ is unramified for all but finitely many places $v$ of $K$. Let $\mathfrak{f}_{\theta}=\prod_{v} \mathfrak{p}^{f_{v}}$. Let $N v=\left|\mathcal{O}_{v} / \mathfrak{p}_{v}\right|$ be the norm of $v$ or equivalently of the prime ideal $\mathfrak{p}_{v}$. We extend the norm map multiplicatively to the group of all fractional ideals of $K$. The conductor $N=N(\theta)$ is defined as $N(\theta)=N_{K / \mathbb{Q}}\left(\mathfrak{f}_{\theta}\right)$.

Let $S_{\infty}$ denote the set of archimedean primes of $K$. Let $r_{1}$ be the number of real places of $K$ and $2 r_{2}$ be the number of complex places of $K$. Denote by $S$ the union of $S_{\infty}$ and the set of ramified primes of $\theta$. Let $|\cdot|$ denote the norm character from the ideles $J_{K}$ of $K$ to $\mathbb{R}^{+}$. Define two idele class characters $\theta_{1}$ and $\theta_{2}$ to be in the same equivalence class if $\theta_{1}=|\cdot|{ }^{s} \theta_{2}$ for some $s \in \mathbb{C}$. It is known that any equivalence class of idele class characters contains a unitary character.

Assume now that $\theta$ is unitary. The $L$-function $L(s, \theta)$ is defined by means of an Euler product in the half plane of convergence. For $s \in \mathbb{C}$ with $\operatorname{Re}(s)>1$ define

$$
L(s, \theta)=\prod_{v \notin S}\left(1-\theta\left(\pi_{v}\right) N v^{-s}\right)^{-1} .
$$

The product converges absolutely for $\operatorname{Re}(s)>1$ and defines an analytic function there.

It was shown by Hecke using the transformation formula for theta functions, and reproven by Tate using Fourier analysis on the adeles, that $L(s, \theta)$ can be analytically continued to the entire complex plane, and that by adding suitable $\Gamma$ factors at the archimedean primes, one can complete the $L$-function, so that it satisfies a functional equation of the appropriate kind [La]. For $v \in S_{\infty}$, write

$$
\theta_{v}(a)=\left(\frac{a}{|a|_{v}}\right)^{m_{v}(\theta)}|a|_{v}^{i n_{v} \phi_{v}(\theta)}
$$

where $n_{v}=1$ if $v$ is real and $n_{v}=2$ if $v$ is complex. If $v$ is real, then $m_{v}(\theta)=0$ or 1 . Define for $v$ archimedean,

$$
s_{v}=s_{v}(\theta)=n_{v}\left(s+i \phi_{v}(\theta)\right)+\left|m_{v}(\theta)\right| .
$$

Let $D_{K}$ denote the discriminant of $K$ over $\mathbb{Q}$ and let $A^{\prime}(\theta)=N(\theta) D_{K}$. Let

$$
\Lambda(s, \theta)=2^{-s r_{2}} \pi^{-s n / 2} A^{\prime}(\theta)^{s / 2} \prod_{v \in S_{\infty}} \Gamma\left(s_{v} / 2\right) L(s, \theta) .
$$

Then the functional equation satisfied by $L(s, \theta)$ is of the form

$$
W(\theta) \Lambda(s, \theta)=\Lambda(1-s, \bar{\theta}),
$$


where $W(\theta)$, the root number of $\theta$, satisfies $|W(\theta)|=1$ and $\bar{\theta}$ is the contragredient of $\theta[\mathrm{La}]$. Let

$$
A=A(\theta)=2^{-r_{2}} \pi^{-n / 2} A^{\prime}(\theta)^{1 / 2} .
$$

It follows from the proof of the functional equation that $L(s, \theta)$ is entire, unless $\theta$ is in the class of the principal character, in which case if $\theta$ is normalised, i.e., if $\sum_{v \in S_{\infty}} n_{v} \phi_{v}(\theta)=0$, then $L(s, \theta)$ has a simple pole at $s=1$. Let $\delta_{\theta}=1$ if $\theta$ is the principal character, and 0 otherwise. It is known that for a normalised character $\theta,[s(s-1)]^{\delta_{\theta}} \Lambda(s, \theta)$ is an entire function of order 1 . Hence if $\theta$ is not in the equivalence class of the principal character, there is a Hadamard product decomposition of the form

$$
\Lambda(s)=a e^{b s} \prod_{\varrho}(1-s / \varrho) e^{s / \varrho},
$$

where $\varrho$ runs over the zeros of $\Lambda(s)$.

We note the variation of the form of the functional equation when we replace $\theta$ by $\theta^{k}$, for a positive integer $k$. It is easy to see that for any finite prime $v, f_{v}\left(\theta^{k}\right) \leq f_{v}(\theta)$. Hence $N\left(\theta^{k}\right) \leq N(\theta)$. It is a fortunate fact that unlike $G L(2)$, the conductors do not grow in the case of $G L(1)$. Moreover, $m_{v}\left(\theta^{k}\right)=k m_{v}(\theta)$ if $v$ is a complex place, and $m_{v}\left(\theta^{k}\right) \equiv k m_{v}(\theta)(\bmod 2)$ if $v$ is a real place, and finally $\phi_{v}\left(\theta^{k}\right)=k \phi_{v}(\theta)$.

We recall that a Dirichlet character is an idele class character of finite order. Thus Dirichlet characters are trivial on the connected components of the completions at the archimedean places. They correspond via class field theory to characters of the Galois group $\operatorname{Gal}(\bar{K} / K)$ of an algebraic closure $\bar{K}$ of $K$. Hence the set of unramified primes for which the local component of a Dirichlet character is the principal character is precisely the set of primes of $K$ which split completely in some cyclic extension of $K$.

We also need to have some knowledge about the zeros of $L(s, \theta)$. Let $N(t)$ denote the number of zeros $\varrho=\beta+i \gamma, 0<\beta<1,|\gamma-| t|| \leq 1$ of $L(s, \theta)$, the density function of the zeros of $L(s, \theta)$ in the critical strip. From the functional equation and the existence of the Hadamard factorisation it can be deduced along the lines of the argument given in [VKM, Lemma 4] that

$$
N(t) \ll \log A+d+\sum_{v \in S_{\infty}} \log \left(n_{v}|t|+\left|\phi_{v}(\theta)\right|+\left|m_{v}(\theta)\right|+4\right),
$$

where $d$ is the degree of $K$ over $\mathbb{Q}$, and where the constant is absolute. In obtaining this estimate, the only difference in our situation is the form of the $\Gamma$ factors, where we need to estimate $\Gamma^{\prime}\left(s_{v} / 2\right) / \Gamma\left(s_{v} / 2\right)$. Since we need the estimate in the region $1<\operatorname{Re}(s)=\sigma \leq 2, s=\sigma+i t$, the required 
estimate is

$$
\left|\frac{\Gamma^{\prime}\left(s_{v}\right)}{\Gamma\left(s_{v}\right)}\right| \ll \log \left(n_{v}|t|+\left|\phi_{v}(\theta)\right|+\left|m_{v}(\theta)\right|+2\right) .
$$

We need to have zero free regions for $L(s, \theta)$. Since we will not need this for the quadratic characters, there are no Siegel zeros to complicate matters. Zero free regions for $L(s, \theta)$ can be constructed following exactly the arguments given in [Dav] for the classical Riemann $\zeta$-function and the complex Dirichlet characters. Again the only difference arises with the estimation of the $\Gamma$ factors, which can be estimated as before. By mimicking the arguments given in [Dav], we obtain

Proposition 1. Let $\theta$ be a unitary idele class character on $K$ not equivalent to a quadratic Dirichlet character. There exists an absolute constant $c>0$, depending only on $K$, such that $L(s, \theta)$ has no zeros $\varrho=\beta+i \gamma$ in the region

$$
\beta>1-c / \log (A(\theta)(|t|+P(\theta)+2)), \quad|t| \geq 0
$$

where

$$
P(\theta)=\sup _{v \in S_{\infty}}\left(\left|\phi_{v}(\theta)\right|+\left|m_{v}(\theta)\right|\right)
$$

2. Explicit formula. The explicit formula method allows one to relate sums of Dirichlet coefficients of suitable $L$-functions to the behavior of the zeros and poles of the $L$-function. We recall the essential features of the method. Our aim is to keep track of the constants involved. For further details about the explicit formula method, we refer to $[\mathrm{LO}],[\mathrm{VKM}],[\mathrm{MR}]$.

The explicit formula method can be applied to a general class of $L$ functions which have a description in terms of an Euler product in some half plane of absolute convergence, an analytic continuation to the entire plane satisfying a suitable functional equation, have a Hadamard factorisation and satisfy the Deligne-Ramanujan estimates, i.e., the eigenvalues of $L(s)$ are of absolute value 1 . For the $L(s, \theta)$ corresponding to a unitary idele class character considered above, the desired analytic properties have been established by Hecke. By $L(s)$, we denote from now onwards an $L$-function of the form $L(s, \theta)$ considered above. We will also assume that $\theta$ is not in the equivalence class of the principal character, or equivalently that $L(s, \theta)$ is an entire function. The numbers $\theta_{v}\left(\pi_{v}\right)$ will be referred to as Hecke eigenvalues or just eigenvalues of $L(s)$. Then the logarithmic derivative of $L(s)$ satisfies

$$
-\frac{L^{\prime}}{L}(s)=\sum_{v, n} \theta_{v}\left(\pi_{v}\right)^{n} \log (N v)(N v)^{-n s},
$$

where $N v$ is the norm of $v$. 
The first step in the explicit formula method is to use the discontinuous integral to approximate the sum of the Hecke eigenvalues of $L(s)$ by means of a "logarithmic integral" for $T \geq 1$ as follows:

$$
\sum_{n \geq 1} \sum_{(N v)^{n}<x} \theta_{v}\left(\pi_{v}\right)^{n} \log (N v)=-\frac{1}{2 \pi i} \int_{c-i T}^{c+i T} \frac{L^{\prime}}{L}(s) x^{s} \frac{d s}{s}+R_{1}(x, T, K, L),
$$

for $c=1+1 / \log x$, where the remainder term satisfies

$$
R_{1}(x, T, K, L) \ll n x(\log x)^{2} / T .
$$

Here by the Vinogradov notation $a \ll b$, we mean that $|a| \leq C|b|$ for some positive absolute constant $C$, which need not be the same at each occurrence.

Next we shift the line of integration leftwards, and integrate over the rectangle $-U \leq \sigma \leq c$ and $|t| \leq T$, where $U=1 / 4+j$, where $j$ is a large positive integer and $T$ is chosen so that $L(\sigma \pm i T) \neq 0$ for any $\sigma$, and $2 \leq T \leq x$. We have

$$
-\frac{1}{2 \pi i} \int_{c-i T}^{c+i T} \frac{L^{\prime}}{L}(s) x^{s} \frac{d s}{s}=S+R_{2}(x, T, U, L, K) .
$$

$S$ denotes the sum of the residues at poles of the integrand inside the rectangle and will be the "main" term. $R_{2}$ is the "remainder" term, the integral over the boundary of the rectangle. By using the functional equation and the Hadamard factorisation, it is possible to show that $R_{2}$ satisfies the following estimate $[\mathrm{VKM}]$ :

$$
\begin{aligned}
R_{2} \ll & \frac{x \log x}{T} \log \left\{A e^{d} \prod_{v \in S_{\infty}}\left(n_{v} T+\left|\phi_{v}(\theta)\right|+\left|m_{v}\right|+5\right)\right\} \\
& +\frac{x^{-U}}{U} T \log \left\{A e^{d} \prod_{v \in S_{\infty}}\left(n_{v}(T+U)+\left|\phi_{v}(\theta)\right|+\left|m_{v}\right|+5\right)\right\} .
\end{aligned}
$$

Note that since we have assumed that $L(s, \theta)$ is entire, the contributions to the terms $R$ and $S$ from the pole on the line $\operatorname{Re}(s)=1$ are absent. The contributions to $S$ are as follows:

(a) Poles coming from the zeros of $L(s)$ in $0<\sigma<1$, which contributes a sum

$$
-\sum_{|\operatorname{Im} \varrho|<T} \frac{x^{\varrho}}{\varrho}
$$

(b) Poles coming from the trivial zeros, which occur at the poles of the $\Gamma$ factors. From the functional equation, it follows that for $x>1$, this is 
bounded by the sum

$$
-\sum_{v \in S_{\infty}} \sum_{m \geq 1} \frac{x^{-\left(2 m+\left|m_{v}\right|\right) / n_{v}}}{\left(2 m+\left|m_{v}\right|\right) / n_{v}} \ll n / x .
$$

Thus we obtain an expression for sums of the eigenvalues of $L$ of the form

$$
\sum_{n \geq 1} \sum_{(N v)^{n}<x} \theta_{v}\left(\pi_{v}\right)^{n} \log (N v)=S_{L}(U, T)+R_{L}(U, T),
$$

where $S$ is a sum over the residues and $R$ is an error term. We now let $U$ go to $\infty$. From the description of $S$ and the fact that $\Lambda(s, \theta)$ is an entire function of order 1 , it can be seen that $S(U, T)$ converges. Using the fact that $\theta$ is unitary, we have

$$
\sum_{(N v)^{n}<x, n \geq 2} \theta_{v}\left(\pi_{v}\right)^{n} \log (N v) \ll d n_{K} x^{1 / 2} .
$$

Putting all these estimates together, we get, for a character $\theta$ not equivalent to the principal character,

$$
\begin{aligned}
& \sum_{N v<x} \theta_{v}\left(\pi_{v}\right) \log (N v)= \\
& \quad-\sum_{|\gamma| \leq T} \frac{x^{\varrho}}{\varrho}+O\left(\frac{d x(\log x)^{2}}{T} \log \left\{A \prod_{v \in S_{\infty}}\left(n_{v} T+\left|\phi_{v}(\theta)\right|+\left|m_{v}(\theta)\right|+5\right)\right\}\right) .
\end{aligned}
$$

By appealing to partial summation we obtain

Proposition 2. Let $\theta$ be a unitary idele class character on $K$ which is not equivalent to the principal character. Then for $2 \leq T \leq x$,

$$
\sum_{N v<x} \theta_{v}\left(\pi_{v}\right)=-\frac{1}{\log x} \sum_{|\gamma| \leq T} \frac{x^{\varrho}}{\varrho}+R(x, L, T),
$$

where $R(x, L, T)$ satisfies

(6) $R(x, L, T) \ll \frac{d x(\log x)}{T} \log \left\{A \prod_{v \in S_{\infty}}\left(n_{v} T+\left|\phi_{v}(\theta)\right|+\left|m_{v}(\theta)\right|+5\right)\right\}$,

and where the implied constant depends only on $K$.

3. Main theorem. We recall the notion of discrepancy $[\mathrm{KN}]$. The discrepancy of a sequence of real numbers is meant to measure the deviation of the sequence from an "ideal" distribution, for example the uniform distribution. Let $\left(x_{j}\right)_{j=1}^{n}$ be a finite sequence of real numbers. For $0 \leq \alpha<\beta \leq 1$, let $A([\alpha, \beta): n)$ be the counting function $\#\left\{j \leq n \mid x_{j} \in[\alpha, \beta)(\bmod 1)\right\}$. 
Define the discrepancy $D_{n}=D_{n}\left(x_{1}, \ldots, x_{n}\right)$ of the sequence as

$$
D_{n}=\sup _{0 \leq \alpha<\beta \leq 1}\left|\frac{A([\alpha, \beta): n)}{n}-(\beta-\alpha)\right| .
$$

If $\omega=\left(x_{i}\right)_{i \geq 1}$ is an infinite sequence of real numbers, the discrepancy $D_{n}(\omega)$ is defined to be the discrepancy of the first $n$ terms of the sequence $\omega$, i.e., $D_{n}(\omega):=D_{n}\left(x_{1}, \ldots, x_{n}\right)$. By a theorem of Weyl, $\omega$ is uniformly distributed modulo 1 if and only if $\lim _{n \rightarrow \infty} D_{n}(\omega)=0$.

For us the importance of the notion of discrepancy comes from a result of Erdős-Turán, which gives an upper bound for the discrepancy of a sequence in terms of exponential sums. The exponential sums which will occur in our situation are of the form $\sum_{N v<x} \theta\left(\pi_{v}\right)$, which can then be estimated by means of the explicit formula method. If it so happens that these exponential sums are "small", then it means that there are cancellations occurring in the sum, or that the terms in the exponential sum are "more evenly distributed". Hence the number of terms with sufficiently small angle should also be relatively small. This heuristic reasoning is made precise with the following result, which can be thought of as giving a quantitative analogue of the theorems on uniform distribution:

Proposition 3 (Erdős-Turán) [KN, p. 112]. For any finite sequence $\left(x_{j}\right)_{j=1}^{n}$ of real numbers and for any positive integer $M$, we have

$$
D_{n} \leq \frac{6}{M+1}+\frac{4}{\pi} \sum_{h=1}^{M}\left(\frac{1}{h}-\frac{1}{M+1}\right)\left|\frac{1}{n} \sum_{j=1}^{n} e^{2 \pi i h x_{j}}\right| .
$$

For a complex number $z$ of modulus 1 , let $\arg (z)$ denote the angle of $z$, i.e., $e^{2 \pi i \arg (z)}=z$, and where we have chosen $\arg (z)$ such that $-1 / 2 \leq$ $\arg (z)<1 / 2$. Denote by $\pi(x)$ the number of primes in $K$ of norm not exceeding $x$, i.e., $\pi(x):=\#\{v \mid N v \leq x\}$. We now state our theorem, which can be considered as an analogue of the prime number theorem, for Hecke characters inequivalent to Dirichlet characters.

THEOREM 1. Let $K$ be a number field and $\theta$ be a unitary idele class character which is not equivalent to a Dirichlet character. Let $S$ be the union of the archimedean places of $K$ and the places of ramification of $\theta$. Let $-1 / 2 \leq$ $\alpha<\beta \leq 1 / 2$. Then there is an absolute constant $c_{1}>0$, depending only on $K$, such that for $x>\max \left(\exp \left(((1-c / 2) \log (A(\theta) P(\theta)))^{2}\right), \exp \left(\log (A(\theta))^{2}\right)\right)$, we have

$$
\begin{aligned}
\#\left\{v \in M_{f}-S \mid N v \leq x, \arg \left(\theta_{v}\left(\pi_{v}\right)\right) \in[\alpha, \beta)\right\} \\
\quad=(\beta-\alpha) \pi(x)+O\left(x \exp \left(-c_{1} \sqrt{\log x}\right)\right),
\end{aligned}
$$

where the implied constant is effectively computable and depends only on $K$. Here $A(\theta)$ and $P(\theta)$ are defined respectively by (1) and (4). In particular, 
the set of primes $v$ of $K$ where the local component $\theta_{v}$ of $\theta$ is the principal character, is of density zero.

Proof. Substituting $x_{v}=\arg \left(\theta_{v}\left(\pi_{v}\right)\right)$ for $v \notin S$, in the discrepancy formula (7), we obtain

$$
\begin{aligned}
& \#\left\{v \notin S \mid N v \leq x, \arg \left(\theta_{v}\left(\pi_{v}\right)\right) \in[\alpha, \beta)\right\} \\
& =(\beta-\alpha) \pi(x) \\
& \quad+O\left(\frac{6 \pi(x)}{M+1}+\frac{4 \pi(x)}{\pi} \sum_{k=1}^{M}\left(\frac{1}{k}-\frac{1}{M+1}\right)\left|\frac{1}{\pi(x)} \sum_{N v \leq x} e^{2 \pi i k x_{v}}\right|\right) \\
& =(\beta-\alpha) \pi(x)+O\left(\frac{\pi(x)}{M}+\sum_{k=1}^{M} \frac{1}{k}\left|\sum_{N v \leq x} \theta_{v}\left(\pi_{v}\right)^{k}\right|\right) .
\end{aligned}
$$

From the explicit formula (5), applied to $L\left(s, \theta^{k}\right)$, we obtain for $2 \leq T_{k} \leq x$,

$$
\left|\sum_{N v \leq x} \theta_{v}\left(\pi_{v}\right)^{k}\right| \ll \frac{1}{\log x}\left|\sum_{|\gamma| \leq T_{k}} \frac{x^{\varrho}}{\varrho}\right|+\left|R\left(x, L_{k}, T_{k}\right)\right|,
$$

where $\varrho$ runs over the zeros of $L\left(s, \theta^{k}\right)$. Since $\theta^{2 k}$ is not equivalent to a principal character, $\theta^{k}$ cannot be equivalent to a quadratic character. Thus we can use the estimates for the zero free region (3) applied to $L\left(s, \theta^{k}\right)$, to obtain

$$
\left|\sum_{|\gamma| \leq T_{k}} \frac{x^{\varrho}}{\varrho}\right| \ll x \exp \left(-\frac{c \log x}{\log \left(A\left(\theta^{k}\right)\left(T_{k}+P\left(\theta^{k}\right)+2\right)\right)}\right) \sum_{j=1}^{T_{k}} \frac{N_{k}(j)}{j},
$$

where $N_{k}(j)$ denotes the density function for the zeros of $L\left(s, \theta^{k}\right)$ in the critical strip. The crucial fact is that $T_{k}$ is at our choice, provided we avoid the ordinates of the zeros of $L\left(s, \theta^{k}\right)$. Let

$$
\begin{aligned}
T_{k} & =\frac{1}{A\left(\theta^{k}\right)} \exp (\sqrt{\log x})-\left(P\left(\theta^{k}\right)+2\right), \\
M & =\exp \left(\frac{c}{2} \sqrt{\log x}\right) .
\end{aligned}
$$

Since $A\left(\theta^{k}\right) \leq A(\theta), P\left(\theta^{k}\right) \leq k P(\theta)$, we see that for $k \leq M$, we have $T_{k} \geq 2$, provided we assume that

$$
\exp (\sqrt{\log x}) \gg A(\theta) P(\theta) \exp \left(\frac{c}{2} \sqrt{\log x}\right) .
$$

This inequality is satisfied if we assume that

$$
x>\exp \left(((1-c / 2) \log (A(\theta) P(\theta)))^{2}\right) .
$$


We have, by (2),

(14) $N_{k}(j) \ll \log A\left(\theta^{k}\right)+d+\sum_{v \in S_{\infty}} \log \left(n_{v} j+\left|\phi_{v}\left(\theta^{k}\right)\right|+\left|m_{v}\left(\theta^{k}\right)\right|+4\right)$ $\ll \log A\left(\theta^{k}\right)+d+\log \left(j+P\left(\theta^{k}\right)+2\right)$.

Hence on substituting (11) for $T_{k}$, we have

(15) $\sum_{j=1}^{T_{k}} \frac{N_{k}(j)}{j} \ll\left(d+\log A\left(\theta^{k}\right)\right) \log T_{k}+\log \left(T_{k}+P\left(\theta^{k}\right)+2\right)^{2} \ll \log x$,

provided we assume that $x>\exp (\log A(\theta))^{2}$. The implied constants in the above inequalities depend only on $K$. Hence from (10), (11) and (15) we obtain

$$
\left|\sum_{|\gamma| \leq T_{k}} \frac{x^{\varrho}}{\varrho}\right| \ll x \log x \exp (-c \sqrt{\log x})\left(1+\frac{k P(\theta)}{\log x}\right),
$$

where $\varrho$ runs over the zeros of $L\left(s, \theta^{k}\right)$.

The remainder term (6) can also be similarly dealt with to obtain

$$
R_{k}=R\left(x, L_{k}, T_{k}\right) \ll \frac{A(\theta) x(\log x)^{d / 2+1}}{\exp (\sqrt{\log x})-A(\theta)\left(P\left(\theta^{k}\right)+2\right)} .
$$

Hence by the explicit formula (9), we have for $x \gg 0$,

$$
\left|\sum_{N v \leq x} \theta_{v}\left(\pi_{v}\right)^{k}\right| \ll x \exp (-c \sqrt{\log x})\left(1+\frac{k P(\theta)}{\log x}\right)+R_{k} .
$$

Substituting this in the discrepancy formula (8) above, we get $\#\left\{v \notin S \mid x_{v} \in[\alpha, \beta)\right\}$

$$
=(\beta-\alpha) \pi(x)+\frac{\pi(x)}{M}+x \exp (-c \sqrt{\log x})\left(\log M+\frac{M P(\theta)}{\log x}\right)+\sum_{k=1}^{M} \frac{R_{k}}{k},
$$

where the implied constant depends only on $K$. For any constants $\alpha>1$, $\beta>0, \alpha>M \beta$, we have

$$
\sum_{k=1}^{M} \frac{1}{k(\alpha-k \beta)} \leq \frac{1}{\alpha}(\log M+\log \alpha) .
$$

This enables us to estimate the remainder term. Substituting $M=$ $\exp ((c / 2) \sqrt{\log x})$ as in (12), we get

$$
\sum_{k=1}^{M} \frac{R_{k}}{k} \ll A(\theta) x(\log x)^{(d+1) / 2+1} \exp (-\sqrt{\log x}) .
$$


Hence we obtain

$$
\#\left\{v \notin S \mid x_{v} \in[\alpha, \beta)\right\}=(\beta-\alpha) \pi(x)+O\left(x \exp \left(-c_{1} \sqrt{\log x}\right),\right.
$$

for some $c_{1}>0$, provided we assume that

$$
x>\max \left(\exp \left(((1-c / 2) \log (A(\theta) P(\theta)))^{2}\right), \exp (\log A(\theta))^{2}\right),
$$

and that proves the theorem.

COROLlaRY 1. Let $K$ be a number field and $\theta$ be an idele class character of infinite order. Let $S$ be the union of the archimedean places of $K$ and the places of ramification of $\theta$. Let $a \in \mathbb{C}^{*}$. Then there exists an absolute constant $c_{-1}>0$, depending only on $K$, such that for

$$
x>\max \left(\exp \left(((1-c / 2) \log (A(\theta) P(\theta)))^{2}\right), \exp (\log A(\theta))^{2}\right),
$$

we have

$$
\#\left\{v \in M_{f}-S \mid N v \leq x, \theta_{v}\left(\pi_{v}\right)=a\right\} \ll x \exp \left(-c_{-1} \sqrt{\log x}\right),
$$

where the implied constant is effectively computable and depends only on $K$.

The corollary follows from the theorem together with the following lemma:

Lemma 1. Let $\theta$ be a character of the form $\chi|\cdot|^{\sigma+i t}$, for some Dirichlet character $\chi$ and some real number $t \neq 0$. Let $a \in \mathbb{C}$. Then there exist at most two rational primes $p_{1}, p_{2}$ with $\left(p_{1} p_{2}, N\right)=1$ such that for some prime $v_{i} \mid p_{i}, \theta_{v_{i}}=a, i=1,2$.

Pro of. Suppose not. Let $p_{3} \neq p_{1}, p_{2}$ be a rational prime satisfying the conclusion of the lemma. Suppose $\chi$ is of order $k$. We have $\left|\pi_{v_{i}}\right|^{k \sigma+k i t}=a^{k}$, $v_{i} \mid p_{i}, i=1,2,3$. From $\left|\pi_{v_{1}} / \pi_{v_{2}}\right|^{k \sigma+k i t}=1$, we obtain $\sigma=0$. Using the ratios of these equations, we deduce that $\left(\log \left|\pi_{v_{1}} / \pi_{v_{2}}\right|\right) /\left(\log \left|\pi_{v_{2}} / \pi_{v_{3}}\right|\right) \in \mathbb{Q}$, which implies that the primes $p_{1}, p_{2}, p_{3}$ are multiplicatively dependent, which is not possible.

In analogy with the conjectures of Lang and Trotter [LT], it is reasonable to expect that the number of primes $v, N v<x$, such that $\theta_{v}=1$, for $\theta$ a character not equivalent to a Dirichlet character, should behave asymptotically as $c \sqrt{x} / \log x$, for some suitable constant $c \geq 0$. The method of proof of the density theorem also proves the following refinement under the Generalised Riemann Hypothesis.

Proposition 4. Suppose $L\left(s, \theta^{k}\right)$ satisfy the Generalised Riemann Hypothesis $(G R H)$, i.e., there are no zeros $\varrho$ of $L\left(s, \theta^{k}\right)$ with $\operatorname{Re}(\varrho)>1 / 2$. Then for $x \gg 0$ depending on $\theta$, we have

$$
\begin{aligned}
\#\left\{v \in M_{f}-S \mid N v \leq x, \arg \left(\theta_{v}\left(\pi_{v}\right)\right)\right. & \in[\alpha, \beta)\} \\
= & (\beta-\alpha) \pi(x)+O\left(x^{1 / 2}(\log x)^{2}\right),
\end{aligned}
$$


where the implied constant is effectively computable and depends only on $K$. In particular,

$$
\#\left\{v \in M_{f}-S \mid N v \leq x, \theta_{v}=1\right\}=O\left(x^{1 / 2}(\log x)^{2}\right) .
$$

Proof. We give a brief outline of the proof. We let $T_{k}=x-P\left(\theta^{k}\right)$ and $M=\sqrt{x}$ in the course of the proof of the above theorem. Under the assumption of GRH, we have from (5),

$$
\begin{aligned}
\sum_{N v<x} \theta_{v}\left(\pi_{v}\right)^{k} & =-\frac{1}{\log x} \sum_{|\gamma| \leq T} \frac{x^{\varrho}}{\varrho}+R\left(x, L, T_{k}\right) \ll \frac{x^{1 / 2}}{\log x} \sum_{j=1}^{T_{k}} \frac{N_{k}(j)}{j} \\
& \ll x^{1 / 2} \log x,
\end{aligned}
$$

provided $x \gg 0$ depending on $\theta$. Substituting this in the discrepancy formula (8), we obtain

$$
\begin{aligned}
\#\left\{v \in M_{f}-S \mid N v \leq x, \arg \right. & \left.\left(\theta_{v}\left(\pi_{v}\right)\right) \in[\alpha, \beta)\right\} \\
= & (\beta-\alpha) \pi(x)+O\left(x^{1 / 2}(\log x) \sum_{k=1}^{M} \frac{1}{k}\right) \\
\ll & (\beta-\alpha) \pi(x)+O\left(x^{1 / 2}(\log x)^{2}\right) .
\end{aligned}
$$

\section{References}

[Dav] H. Davenport, Multiplicative Number Theory, 2nd ed., Grad. Texts in Math. 74, Springer, New York, 1980.

[He] E. Hecke, Eine neue Art von Zetafunktionen und ihre Beziehungen zur Verteilung der Primzahlen, Zweite Mitteilung, Math. Z. 6 (1920), 11-51; reprinted in Mathematische Werke, Vandenhoeck \& Ruprecht, Göttingen, 1959, 249-289.

[KN] L. Kuipers and H. Niederreiter, Uniform Distribution of Sequences, Wiley, New York, 1974.

[LO] J. Lagarias and A. M. Odlyzko, Effective versions of the Chebotarev density theorem, in: Algebraic Number Fields, A. Fröhlich (ed.), Academic Press, New York, 1977, 409-464.

[La] S. Lang, Algebraic Number Theory, Grad. Texts in Math. 110, Springer, New York, 1986.

[LT] S. Lang and H. Trotter, Frobenius Distributions in $G L_{2}$ Extensions, Lecture Notes in Math. 504, Springer, New York, 1976.

[MR] M. R. Murty and C. S. Rajan, Stronger multiplicity one theorems for forms of general type on $G L_{2}$, in: Analytic Number Theory, Proc. Conf. in Honor of Heini Halberstam, Vol. 2, B. C. Berndt, H. G. Diamond and A. J. Hildebrand (eds.), Birkhäuser, Boston, 1996, 669-683. 
[VKM] V. K. Murty, Explicit formulae and the Lang-Trotter conjecture, Rocky Mountain J. Math. 15 (1985), 535-551.

School of Mathematics

Tata Institute of Fundamental Research

Homi Bhabha Road

Mumbai, 400 005, India

E-mail: rajan@math.tifr.res.in

Received on 19.8.1997

and in revised form on 27.1.1998 